\title{
Bioremediation of Silver and Recovery of AgNPs for the Fabrication of AgNPs Functionalized Antibacterial Polycaprolactone Membrane
}

\author{
Fiaz Ahmad, Noreen Ashraf, Yin Da-Chuan \\ Institute for Special Environmental Biophysics, Key Laboratory for Space Bioscience and Biotechnology \\ School of Life Sciences, Northwestern Polytechnical University \\ Xi' an 710072, Shaanxi, PR China \\ fiaz.a@mail.nwpu.edu.cn; noreenashrafnpu@yahoo.com; yindc@nwpu.edu.cn
}

\section{Extended Abstract}

Heavy metals denote a group of hazardous environmental contaminants due to their toxic effects on human health in concentrations higher than the permissible limits which cause widespread concerns [1]. Silver is a precious as well as toxic heavy metal; hence, its remediation and recovery are essential for environmental sustainability and biomedical applications. So far, conventional methods, i.e., precipitation, coagulation, and ion exchange are less efficient at low metal concentrations and expensive in comparison with bioremediation of heavy metal pollutants. Therefore, the isolation of heavy metal resistant bacteria from metal contaminated sites and their use for the remediation and recovery of metals is documented [2,3]. However, there are very few or no reports of simultaneous remediation and recovery of silver in the form of silver nanoparticles (AgNPs). Due to the chemical stability, bactericidal and optical properties of AgNPs, they are widely used in many biomedical and environmental applications [4].

In this study, we have isolated three potential bacterial strains from silver mining site (Zhashui, Shaanxi Province, China) for their potential use in silver remediation and its recovery in the form of AgNPs. The synthesized biogenic AgNPs were characterized and utilized to fabricate antibacterial membrane filter using nanofibers of polycaprolactone (PCL) functionalized with AgNPs by dissolved electrospinning method for their potential use in biomedical filtration devices.

The novel bacterial strains were isolated from soil and water showing a long history of silver contamination and identified using restriction fragment length polymorphism, and through 16S DNA sequencing followed by NCBI Blast analysis. Based on preliminary experimental results, one (SMP1) out of three bacterial strains was selected for further studies. The detection and quantification of silver removal and recovery of AgNPs from spiked aqueous medium were measured by using atomic absorption spectroscopy (AAS). Synthesized AgNPs were purified through centrifugation and by the application of external voltage directly from the reaction mixture. Biogenic AgNPs were characterized by using UVspectrophotometer, dynamic light scattering (DLS), SEM and TEM. Diethyl ether was used to dissolve PCL followed by mixing of AgNPs to prepare AgNPs and PCL (AgNPs-PCL) composite, and an antibacterial AgNPs-PCL filter membrane was fabricated by using electrospinning technique. Disc diffusion method was used to access the antibacterial potential of the developed membrane filter.

Results obtained showed that potentially all three isolated bacterial strains identified as Enterobacter cloacae (SMP1), Cupriavidus necator (SMP2), and Bacillus megaterium (SMP3) were highly efficient with more than 95\% silver remediation efficiency. However, SMP1 was selected for further studies based on its quick and highest remediation of silver as well as high efficiency of AgNPs recovery. The remediation efficiency of AgNPs was 100\% in less than 1 hour. Strain (SMP1) was able to synthesize 10 to $20 \mathrm{~nm}$ sized spherical and partially cubic AgNPs on their cell surface as well as extracellularly. The fabricated AgNP-PCL membrane filter showed significant bactericidal potential against Pseudomonas aeruginosa and Escherichia coli with considerable reusability.

Potentially this approach could be implemented for the remediation of different heavy metal contaminated sites and recovered nanoparticles that can be used in various biomedical and environmental applications.

\section{References}

[1] A.T. Jan, M. Azam, K. Siddiqui, A. Ali, I. Choi, Q.M.R. Haq, "Heavy metals and human health: Mechanistic insight into toxicity and counter defense system of antioxidants," Int. J. Mol. Sci., vol. 16, no. 12, pp. 26183, 2015. 
[2] Q. Jing, M. Zhang, X. Liu, Y. Li, Z. Wang, J. Wen, "Bench-scale microbial remediation of the model acid mine drainage: Effects of nutrients and microbes on the source bioremediation," Int. Biodeterior. Biodegrad., vol. 128, pp. 117-121, 2018.

[3] Z. Teng, W. Shao, K. Zhang, Y. Huo, M. Li, "Characterization of phosphate solubilizing bacteria isolated from heavy metal contaminated soils and their potential for lead immobilization," J. Environ. Manage., vol. 231, pp. 189-197, 2019.

[4] B. Momin, S. Rahman, N. Jha, U.S. Annapure, "Valorization of mutant Bacillus licheniformis M09 supernatant for green synthesis of silver nanoparticles: photocatalytic dye degradation, antibacterial activity, and cytotoxicity," Bioprocess Biosyst. Eng., pp. 1-13, 2019. 